\title{
Existence of Gauss Interpolation Formulas for the One-Dimensional Heat Equation
}

\author{
By David L. Barrow
}

\begin{abstract}
Let $C=\{(x(s), t(s)): a \leqslant s \leqslant b\}$ be a Jordan arc in the $x$-t plane satisfying $(x(a), t(a))=\left(a, t_{*}\right),(x(b), t(b))=\left(b, t_{*}\right)$, and $t(s)<t_{*}$ when $a<s<b$. Let $a<$ $x_{*}<b$. We prove the existence of Gauss interpolation formulas for $C$ and the point $\left(x_{*}, t_{*}\right)$, for solutions $u$ of the one-dimensional heat equation, $u_{t}=u_{x x}$. Such formulas approximate $u\left(x_{*}, t_{*}\right)$ in terms of a linear combination of its values on $C$. The formulas are characterized by the requirement that they are exact for as many basis functions (the heat polynomials) as possible.
\end{abstract}

1. Introduction. We consider the problem of solving the one-dimensional heat equation

$$
\partial u / \partial t=\partial^{2} u / \partial x^{2}
$$

when $u$ is known on an initial-boundary curve $C$ in the $x$ - $t$ plane. Specifically, we are interested in formulas of the form

$$
u\left(x_{*}, t_{*}\right) \cong \sum_{i=1}^{N} A_{i} u\left(x_{i}, t_{i}\right),
$$

where the points $\left(x_{i}, t_{i}\right)$ are on $C$ and the weights $A_{i}$ are positive. The formulas are of Gauss type in the sense that the points and weights are chosen to give zero error for a maximum number of basis functions (approximately $2 N$ of them). The basis functions used are the heat polynomials of Appell (see Section 3).

Shriver [10] studied such formulas, as well as generalizations to the $n$-dimensional heat equation. He proved the existence of the formulas in certain cases, and described some numerical results in obtaining and using them. In [12] and [13], Stroud discussed similar formulas for the Dirichlet and Neumann problems. Barrow and Stroud [2] proved the existence of Gauss formulas for the two-dimensional Dirichlet problem using topological degree. This result was announced in [14]. Davis and Wilson [5] proved the existence of interpolation formulas for solutions to second order elliptic equations over bounded domains in $R^{n}$. However, these were $N$-point formulas exact for $N$ basis functions, and hence not of Gauss type.

In Section 2 we introduce some definitions and notation, present some results from the theory of Tchebycheff systems, and then state the main result of this paper, Theorem 1. Section 3 is concerned with the heat polynomials and some of their properties. In Section 4 we introduce some basic properties of finite-dimensional topological degree and use them to complete the proof of Theorem 1. Section 5 contains a numerical example from Shriver's thesis.

Received December 23, 1974; revised March 27, 1975.

AMS (MOS) subject classifications (1970). Primary 65N99; Secondary 35K05. 


\section{Definitions and Statement of Existence Theorem.}

Definition 1. Let $t_{*}, a, b$ be fixed numbers with $a<b$. Let $C=\{(x(s), t(s))$ : $a<s<b\}$ be a continuous, nonselfintersecting curve (i.e., a Jordan arc) in the $x-t$ plane satisfying $(x(a), t(a))=\left(a, t_{*}\right),(x(b), t(b))=\left(b, t_{*}\right)$, and $t(s)<t_{*}$ when $a<s$ $<b$. Then $C$ will be called an initial-boundary curve or data curve, and we let $C$ denote the collection of all such curves.

Definition 2. For $k$ a nonnegative integer, let $u_{k}$ be the polynomial solution to Eq. (1) defined by

$$
u_{k}(x, t)=k ! \sum_{j=0}^{[k / 2]} \frac{x^{k-2 j} t^{j}}{(k-2 j) ! j !},
$$

where $[a]$ means the greatest integer less than or equal to $a$. For $n$ a nonnegative integer, let $H_{n}$ denote the real vector space spanned by the $\left\{u_{k}\right\}_{k=0}^{n}$. If $p=$ $\Sigma_{k=0}^{n} a_{k} u_{k}$ with $a_{n} \neq 0$, we say $p$ is a heat polynomial of degree $n$.

Definition 3. Assume $w_{0}(s), w_{1}(s), \ldots, w_{n}(s)$ are continuous functions defined on an interval $a \leqslant s \leqslant b$. These functions are said to be a Tchebycheff system if the determinant

$$
\left|\begin{array}{ll}
w_{0}\left(s_{0}\right) & w_{0}\left(s_{1}\right) \cdots w_{0}\left(s_{n}\right) \\
w_{1}\left(s_{0}\right) & w_{1}\left(s_{1}\right) \cdots w_{1}\left(s_{n}\right) \\
\cdots & \ldots \\
w_{n}\left(s_{0}\right) & w_{n}\left(s_{1}\right) \cdots w_{n}\left(s_{n}\right)
\end{array}\right|
$$

is strictly positive whenever $s_{0}, s_{1}, \ldots, s_{n}$ satisfy $a \leqslant s_{0}<s_{1}<\cdots<s_{n} \leqslant b$.

Definition 4. Let $\left\{w_{k}(s)\right\}_{k=0}^{n}$ be a Tchebycheff system. The moment space $M_{n+1}$ associated with this system is the subset of Euclidean space $R^{n+1}$ determined as follows:

$$
M_{n+1}=\left\{c=\left(c_{0}, c_{1}, \ldots, c_{n}\right): c_{i}=\int_{a}^{b} w_{i}(s) d \sigma(s)\right\}
$$

where $\sigma(s)$ traverses the set of all nondecreasing right continuous bounded functions.

We now discuss some basic properties of Tchebycheff systems (cf. [6, Chapter 2]). The moment space $M_{n+1}$ can be identified with the set of nonnegative linear functionals on $C_{n}[a, b]$, the real vector space spanned by the $\left\{w_{k}(s)\right\}_{k=0}^{n}$. The interior of $M_{n+1}$ corresponds to the set of strictly positive linear functionals. (A linear functional $L$ is nonnegative if $p \in C_{n}[a, b]$ and $p(s) \geqslant 0$ imply $L(p) \geqslant 0$; it is strictly positive if $p(s) \geqslant 0$ and $p(s) \not \equiv 0$ imply $L(p)>0$.) $L$ can be represented in $M_{n+1}$ by the vector $\left(L w_{0}, \ldots, L w_{n}\right)$. Each point $c=\left(c_{0}, \ldots, c_{n}\right) \in M_{n+1}$ can be represented in the form

$$
c_{i}=\sum_{j=1}^{p} A_{j} w_{i}\left(s_{j}\right), \quad i=0, \ldots, n,
$$

where each $A_{j}>0$ and $a \leqslant s_{1}<s_{2}<\cdots<s_{p} \leqslant b$. The index of the representation 
is defined to be the sum obtained by counting one for each $a<s_{j}<b$ and one-half for $s_{j}=a$ or $b$.

The following result is proved in [6,pp. 44-47]: Let $c$ be an interior point of $M_{n+1}$. Then $c$ has precisely two representations of the form (4) which have index $(n+1) / 2$. One of these involves the point $b$, and the other does not. Furthermore, there is no representation for $c$ having a smaller index. Theorem 1 , below, is analogous to this result; it is, in fact, a direct consequence of it for certain data curves $C$. We first give two more definitions.

Definition 5. Let $C \in C$ and let $a<x_{*}<b$. For an interpolation formula (2) with distinct points $\left(x_{i}, t_{i}\right) \in C$, we define its index to be the number obtained by summing over the points in the formula, counting one if $t_{i}<t_{*}$ and one-half if $t_{i}=t_{*}$.

Definition 6. Let $C$ and $x_{*}$ be as in Definition 5. If a formula (2) with points on $C$ is exact for all $u \in H_{n}$ and it has index $(n+1) / 2$, we say it is a Gauss interpolation formula (for $C,\left(x_{*}, t_{*}\right)$, and $\left.H_{n}\right)$.

THEOREM 1. Let $C$ be a curve in $C$, and let $n$ be a positive integer. Let $a<x_{*}$ $<$ b. Then

(i) there are at least two Gauss interpolation formulas for $C,\left(x_{*}, t_{*}\right)$, and $H_{n}$; one of these involves the point $\left(b, t_{*}\right)$ and the other does not;

(ii) the vectors $\left(u_{0}\left(x_{i}, t_{i}\right), u_{1}\left(x_{i}, t_{i}\right), \ldots, u_{n}\left(x_{i}, t_{i}\right)\right), i=1, \ldots, N$, where the $\left(x_{i}, t_{i}\right)$ are the points in a Gauss formula, are linearly independent;

(iii) there is no interpolation formula for $C,\left(x_{*}, t_{*}\right)$, and $H_{n}$ having index smaller than $(n+1) / 2$.

3. The Heat Polynomials. In the paper [1], Appell introduced the heat polynomials $u_{k}(x, t)$ (Eq. (3)) which he defined as the coefficients of $z^{n} / n$ ! in the power series expansion of $\exp \left(z x+z^{2} t\right)$, i.e.,

$$
e^{z x+z^{2} t}=\sum_{k=0}^{\infty} u_{k}(x, t) z^{n} / n !
$$

Each $u_{k}$ is a solution to (1), and can in fact be derived as the polynomial solution to (1) satisfying the initial data $u_{k}(x, 0)=x^{k}$, by a Taylor's series expansion in $t$.

In [9] Rosenbloom and Widder considered expansions of solutions of (1) in terms of heat polynomials. Widder [15] showed that the set $\left\{u_{k}\right\}_{k=0}^{\infty}$ is complete, in the maximum norm, in the space of solutions to (1) which are analytic in a neighborhood of the origin; i.e., if $u(x, t)$ is a solution to Eq. (1) which is analytic for $|x| \leqslant c$, $|t| \leqslant c$, then $u$ can be approximated arbitrarily closely by a finite linear combination of the functions $\left\{u_{k}\right\}_{k=0}^{\infty}$. Colton [3, Lemma 2.1] showed that the $\left\{u_{k}\right\}_{k=0}^{\infty}$ are complete for the space of strong solutions to Eq. (1) in a region $R=\{(x, t):|x| \leqslant 1$, $0 \leqslant t \leqslant T\}$ which are continuous on $\bar{R}$. In [4], he extended this result to regions of the form $\left\{(x, t): 0 \leqslant t \leqslant T, s_{1}(t) \leqslant x \leqslant s_{2}(t)\right\}$ where $x=s_{1}(t)$ and $x=s_{2}(t)$ are analytic arcs satisfying $s_{1}(t)<s_{2}(t)$ for $0 \leqslant t \leqslant T$. It follows easily that for such regions (and their translates), a sequence of interpolates (2) will converge, as $N \rightarrow \infty$, to $u\left(x_{*}, t_{*}\right)$ for any strong solution $u$ continuous on $\bar{R}$. 
In this section we develop the properties of heat polynomials required in the proof of Theorem 1, the most important being Lemmas 5 and 6.

LEMMA 1. If $p \in H_{n}$, then

$$
p(x, t)=\sum_{k=0}^{n} \frac{\partial^{k} p\left(x_{0}, t_{0}\right)}{\partial x^{k}} \frac{u_{k}\left(x-x_{0}, t-t_{0}\right)}{k !} .
$$

Proof. Both functions are polynomial solutions to the heat equation which are equal on the line $t=t_{0}$, as follows from a Taylor's series expansion in $x$ of $p\left(x, t_{0}\right)$. Hence, by a Taylor's series expansion in $t$, they are equal everywhere.

LEMMA 2. The functions $u_{k}(x, t)$ satisfy

$$
\frac{\partial u_{k}(x, t)}{\partial x}=k u_{k-1}(x, t), \quad k=1,2, \ldots,
$$

and

$$
u_{k}(x, t)=x u_{k-1}(x, t)+2 t(k-1) u_{k-2}(x, t), \quad k=2,3, \ldots .
$$

Proof. Both functions in (i) are polynomial solutions to Eq. (1) which equal $k x^{k-1}$ when $t=0$, and so they are equal everywhere. Similar reasoning proves (ii), where (i) is used to show that the right side of (ii) satisfies the heat equation.

We introduce the following notation:

$$
f_{k}(c)=u_{k}\left(x, c x^{2}\right) / x^{k}, \quad k=0,1,2, \ldots,
$$

where $c$ is any real number. Thus, $f_{0}(c)=1, f_{1}(c)=1, f_{2}(c)=1+2 c, f_{3}(c)=1+$ $6 c$, etc. The recursion relation in Lemma 2 implies

$$
f_{k}(c)=f_{k-1}(c)+2 c(k-1) f_{k-2}(c), \quad k=2,3, \ldots .
$$

LEMMA 3. There is a sequence $\left\{c_{k}\right\}_{k=1}^{\infty}$ satisfying $-1=c_{1}<c_{2}<\cdots<c_{k}$ $<\cdots<0$, such that for $k=2,3, \ldots$

(i) $f_{k}\left(c_{k}\right)=0$ and $f_{k}(c)>0$ for $c_{k}<c \leqslant 0$.

(ii) $f_{k}\left(c_{k-1}\right)<0$.

Proof. Let $c_{2}=-1 / 2$; then (i) $)_{2}$ and (ii) $)_{2}$ are clear. Now let $k>2$, and suppose (i) $n$ and (ii) $n$ are true for $n<k, n \geqslant 2$. Then by Eq. (5),

$$
f_{k}\left(c_{k-1}\right)=f_{k-1}\left(c_{k-1}\right)+2 c_{k-1}(k-1) f_{k-2}\left(c_{k-1}\right)<0,
$$

by the induction hypothesis. This proves (ii) $)_{k}$. Now since $f_{k}(0)=1$, there is a $c_{k}>$ $c_{k-1}$ satisfying (i) $)_{k}$, and the lemma follows by induction.

Lemma 4. Let $p \in H_{n}$ be nonzero and let $Z\left(I_{\epsilon}, t\right)$ be the number of distinct zeroes of $p(x, t)$ in the interval $I_{\epsilon}=\{-\epsilon<x<\epsilon\}$, for a given $\epsilon>0$. Assume $p(0,0)$ $=0$. Then there are positive numbers $\epsilon$ and $\delta$ such that exactly one of the following is true:

$$
\begin{aligned}
Z\left(I_{\epsilon}, t\right) & =1, & & -\delta<t<\delta, \\
Z\left(I_{\epsilon}, t\right) & =0, & & 0<t<\delta, \\
& =1, & & t=0, \\
& \geqslant 2, & & -\delta<t<0,
\end{aligned}
$$




$$
\begin{aligned}
Z\left(I_{\epsilon}, t\right) & =1, \quad 0 \leqslant t<\delta, \\
& \geqslant 3, \quad-\delta<t<0 .
\end{aligned}
$$

Proof. Case (i), $\partial p(0,0) / \partial x \neq 0$. The Implicit Function Theorem implies there is a unique solution $x=x(t)$ to $p(x, t)=0$ in the region $|x|<\epsilon,|t|<\delta$, for sufficiently small $\epsilon, \delta$, and (i) is proved.

For cases (ii) and (iii), we assume $\partial p(0,0) / \partial x=0$. Then by Lemma $1, p(x, t)$ $=\Sigma_{k=N}^{n} b_{k} u_{k}(x, t)$, where $N \geqslant 2$ and $b_{N} \neq 0$. Without loss of generality, we take $b_{N}=1$.

Case (ii), $N=2 m$. Then $p(x, 0)=x^{2 m}+b_{N+1} x^{N+1}+\cdots$, so there are $\epsilon>0, \delta_{1}>0$ such that $p(x, 0)>0$ for $0<|x| \leqslant \epsilon$ and $p( \pm \epsilon, t)>0$ for $|t| \leqslant \delta_{1}$. It follows from the maximum principle [8, p. 168] that $p(x, t)>0$ for $|x|<\epsilon, 0<$ $t \leqslant \delta_{1}$. For $t<0$, we consider $p(x, t)$ on a curve $t=c x^{2}, c<0$. On such a curve, $p(x, t)=p\left(x, c x^{2}\right)=x^{2 m} f_{N}(c)+b_{N+1} x^{N+1} f_{N+1}(c)+\cdots$. Hence, for $|x|$ small and nonzero, the sign of $p\left(x, c x^{2}\right)$ is equal to the sign of $f_{N}(c)$. By Lemma 3 , there is a $c_{-}<0$ such that $f_{N}\left(c_{-}\right)<0$. We can therefore find a $\delta \leqslant \delta_{1}$ so small that for $-\delta<t<0, p(x, t)$ changes sign at least twice on $I_{\epsilon}$, and hence has at least two zeroes there. This proves (ii).

Case (iii), $N=2 m+1$. We first apply the results of (ii) above to $\partial p(x, t) / \partial x$ to obtain $\epsilon_{1}>0, \delta_{1}>0$ such that $\partial p(x, t) / \partial x>0$ for $|x|<\epsilon_{1}$ and $0<t<\delta_{1}$. Now since $p(x, 0)=x^{2 m+1}+b_{N+1} x^{N+1}+\cdots$, there are $0<\epsilon \leqslant \epsilon_{1}, 0<\delta_{2}<\delta_{1}$ such that $p(x, 0)<0$ for $-\epsilon<x<0, p(x, 0)>0$ for $0<x<\epsilon, p(-\epsilon, t)<0$ for $|t|<\delta_{2}$ and $p(\epsilon, t)>0$ for $|t|<\delta_{2}$. It follows that $p(x, t)$ has exactly one zero on $I_{\epsilon}$ when $0 \leqslant t<\delta_{2}$. For $t<0$, there is a $c_{-}<0$, as before, such that $f_{N}\left(c_{-}\right)<0$, and we deduce that for some $0<\delta \leqslant \delta_{2}, p(x, t)$ has at least three sign changes on $I_{\epsilon}$, and hence at least three zeroes there, when $-\delta<t<0$. This completes the proof of the lemma.

Remark. If $p \in H_{n}$ and $p\left(x_{0}, t_{0}\right)=0$, we can use Lemma 1 and a change of variables to prove that a corresponding result holds near $\left(x_{0}, t_{0}\right)$.

Lemma 5. Let $z_{i}=\left(x_{i}, t_{i}\right), i=0,1, \ldots, k+m$ be distinct points such that $t_{i}=t_{0}, i=1, \ldots, k$ and $t_{i}<t_{0}, i=k+1, \ldots, k+m$. Let $n=k+2 m$. Then the point evaluation linear functionals $f_{z_{i}}$, defined by $f_{z_{i}}(p)=p\left(z_{i}\right)$, are linearly independent on $H_{n}$.

Proof. The conclusion of the lemma is clearly equivalent to the statement that the vectors $\left(u_{0}\left(z_{i}\right), u_{1}\left(z_{i}\right), \ldots, u_{n}\left(z_{i}\right)\right), i=0, \ldots, k+m$ are linearly independent. Suppose that this set is dependent. Then so also is the set obtained by adjoining the vectors $\left(\partial u_{0}\left(z_{i}\right) / \partial x, \ldots, \partial u_{n}\left(z_{i}\right) / \partial x\right), i=k+1, \ldots, k+m$. Hence there is a nonzero $p \in H_{n}$ such that $p\left(z_{i}\right)=0, i=0,1, \ldots, k+m$ and $\partial p\left(z_{i}\right) / \partial x=0, i=k+1$, $\ldots, k+m$. We will show that this is impossible.

Let $T>0$ be such that $\left|t_{i}\right|<T, i=0,1, \ldots, k+m$. For each $t$, let $Z(t)$ be the number of distinct zeroes of $p(x, t)$. We claim that $Z(-T) \geqslant n+1$; this will imply that $p(x,-T) \equiv 0$, from which $p(x, t) \equiv 0$ follows.

Let $p(x, t)$ have degree $r \leqslant n$. Then $p(x, t)=a_{r} x^{r}+R(x, t)$, where $R(x, t)$ has degree less than $r$. Hence, there is some $X>0$ such that $p(x, t) \neq 0$ for $|t| \leqslant T$ and 
$|x| \geqslant X$. For $|\tau|<T$, let $x=y_{1}, y_{2}, \ldots, y_{j}$ be the zeroes of $p(x, \tau)$. For $\epsilon>0$, let $K$ be the compact set

$$
K=[-X, X] \backslash \bigcup_{i=1}^{j} I_{\epsilon}+y_{i}
$$

where $I_{\epsilon}+y_{i}$ is the interval $\left(y_{i}-\epsilon, y_{i}+\epsilon\right)$. Then $K$ is a positive distance from the zero set of $p(x, t)$, so there is a $\delta>0$ such that

$$
Z(t)=\sum_{i=1}^{j} Z\left(I_{\epsilon}+y_{i}, t\right),
$$

when $|t-\tau|<\delta$. Let $j_{1}, j_{2}, j_{3}$ be the number of zeroes of $p(x, \tau)$ of type (i), (ii), or (iii), respectively, of Lemma 4 . Then by that lemma, and the remark following it, for $\delta$ sufficiently small, $Z(t)=j_{1}+j_{3}$ for $\tau<t<t+\delta, Z(t)=j_{1}+j_{2}+j_{3}$ for $t=\tau$, and $Z(t) \geqslant j_{1}+2 j_{2}+3 j_{3}$ for $\tau-\delta<t<\tau$. Hence, as $t$ decreases, $Z(t)$ is nondecreasing, and it increases by at least two due to each of the $m$ points where $t_{i}<t_{0}$. Since $Z\left(t_{0}\right) \geqslant k+1, Z(-T) \geqslant k+1+2 m=n+1$, as claimed.

Corollary. A formula of the form (2) with points on a curve $C \in C$ and which is exact for all $u \in H_{n}$ cannot have index smaller than $(n+1) / 2$.

Proof. Let $I$ be the index of the formula. If the formula has $k$ points, $k=0,1$, or 2 , each contributing one-half to the index and $m$ points each contributing one then $I=m+k / 2$. If $n=k+2 m$ (or is larger), Lemma 5 implies that the functional of evaluation at $\left(x_{*}, t_{*}\right)$ cannot be a linear combination of the functionals of evaluation at the points in the formula. Hence, $n<2 I$, or $I \geqslant(n+1) / 2$.

LEMMA 6. Let $\Gamma$ be the parabola

$$
x=s, \quad t=t_{0}+c\left(s-x_{0}\right)^{2}, \quad c \geqslant 0, \quad s_{1} \leqslant s \leqslant s_{2} .
$$

Then the functions $\left\{u_{k}\right\}_{k=0}^{n}$, when restricted to $\Gamma$, form an extended Tchebycheff system of arbitrary order (see Karlin and Studden [6,p. 6]).

Proof. Let $v_{k}(s)=u_{k}\left(s, t_{0}+c\left(s-x_{0}\right)^{2}\right)$, and suppose that $p(s)=$ $\Sigma_{k=0}^{n} a_{k} v_{k}(s)$ has at least $n+1$ zeroes, counting multiplicities, on $s_{1} \leqslant s \leqslant s_{2}$. Let

$$
q(x, t)=\sum_{k=0}^{n} a_{k} u_{k}\left(x+x_{0}, t+t_{0}\right)
$$

Then $q \in H_{n}$, and by Lemma 1 ,

$$
q(x, t)=\sum_{k=0}^{n} \frac{\partial^{k} q(0,0)}{\partial x^{k}} \frac{u_{k}(x, t)}{k !}
$$

Hence, the polynomial

$$
q\left(s, c s^{2}\right)=\sum_{k=0}^{n} \frac{\partial^{k} q(0,0)}{\partial x^{k}} \frac{f_{k}(c) s^{k}}{k !}=p\left(s+x_{0}\right)
$$

has at least $n+1$ zeroes on $s_{1}-x_{0} \leqslant s \leqslant s_{2}-x_{0}$. Therefore, $\partial^{k} q(0,0) / \partial x^{k}=0$, $k=0,1, \ldots, n$ (since $\left.f_{k}(c)>0\right)$, and this shows $p(s) \equiv 0$.

4. Proof of Theorem 1. To complete the proof, we shall need the concept of the finite-dimensional degree of a mapping (see Schwartz [11], or Ortega and 
Rheinboldt [7]). Let $D \subset R^{N}$ be an open bounded set, and let $F: \bar{D} \rightarrow R^{N}$ be continuous. If $q \in R^{N}$ and $q \notin F(\partial D)$, then the degree $F$ with respect to $D$ and $q$ is defined, and will be denoted by $\operatorname{deg}(F, D, q)$. The following are some basic properties of the degree:

(i) If $F \in C^{1}(D)$ and $J F\left(x_{i}\right) \neq 0$ whenever $F\left(x_{i}\right)=q$ (where $J F$ is the Jacobian of $F$ ), then there are a finite number of points $x_{i} \in D$ where $F\left(x_{i}\right)=q$ and $\operatorname{deg}(F, D, q)=\Sigma_{i} \operatorname{sgn} J F\left(x_{i}\right)$.

(ii) If $\operatorname{deg}(F, D, q) \neq 0$, there is at least one point $x \in D$ such that $F(x)=q$.

(iii) Let $F(x, \lambda)$ be continuous on $\bar{D} \times I$, where $\lambda \in I=[0,1]$. Let $F(x, \lambda) \neq q$ for $x \in \partial D$ and $\lambda \in I$. Then $\operatorname{deg}(F(\cdot, \lambda), D, q)$ is constant for $\lambda \in I$.

Let $C \in \mathcal{C}$ be as in the statement of Theorem 1, and let $C^{\prime} \in \mathcal{C}$ be a parabola as in Lemma 6, of the form $t=\sigma(s)=t_{0}+c\left(s-x_{0}\right)^{2}, a \leqslant s \leqslant b, c \geqslant 0$. We will use the theory of Tchebycheff systems to prove the theorem for $C^{\prime}$, and then use degree theory to prove it for $C$.

Let $v_{k}(s)=u_{k}(s, \sigma(s)), k=0,1, \ldots, n$. Then $\left\{v_{k}(s)\right\}_{k=0}^{n}$ is an extended Tchebycheff system by Lemma 6 . Let

$$
q=\left(u_{0}\left(x_{*}, t_{*}\right), u_{1}\left(x_{*}, t_{*}\right), \ldots, u_{n}\left(x_{*}, t_{*}\right)\right) .
$$

LEMMA 7. The point $q$ is an interior point of the moment space $M_{n+1}$ determined by the functions $\left\{v_{k}(s)\right\}_{k=0}^{n}$.

Proof. Let $V_{n}$ be the span of $\left\{v_{k}(s)\right\}_{k=0}^{n}$. There is a natural isomorphism between $H_{n}$ and $V_{n}$ determined by restriction to $C^{\prime}$, i.e.,

$$
p(x, t)=\sum_{k=0}^{n} a_{k} u_{k}(x, t) \rightarrow \sum_{k=0}^{n} a_{k} v_{k}(s) \equiv p(s) .
$$

Let $L$ be the linear functional defined on $V_{n}$ by $L p=p\left(x_{*}, t_{*}\right)$. Then $q=\left(L u_{0}, L u_{1}\right.$, $\left.\ldots, L u_{n}\right) \in \operatorname{Int} M_{n+1}$ if $L$ is positive. But this follows from the maximum principle for solutions to the heat equation on the region bounded by $C^{\prime}$ and $t=t_{*}$ (see [8, p. 168]), and the lemma is proved.

We now let $C_{\lambda}, 0 \leqslant \lambda \leqslant 1$ be a continuous deformation of $C^{\prime}$ into $C$ such that each $C_{\lambda} \in C$. Let $C_{\lambda}$ be given parametrically by $\{(x(s, \lambda), t(s, \lambda)): a \leqslant s \leqslant b\}$; with $C_{0}=C^{\prime}$ and $C_{1}=C$. Let

$$
v_{k}(s, \lambda)=u_{k}(x(s, \lambda), t(s, \lambda)) \text {. }
$$

To proceed further, we must consider separate cases.

Case (i), $n=2 m$ and the formula does not involve the point $\left(b, t_{*}\right)$. Let $D$ be the subset of $R^{n+1}$ defined by

$$
\begin{array}{r}
D=\left\{x=\left(A_{0}, A_{1}, \ldots, A_{m}, s_{1}, \ldots, s_{m}\right): 0<A_{i}<1, i=0, \ldots, m\right. \\
\text { and } \left.a=s_{0}<s_{1}<s_{2}<\cdots<s_{m}<b\right\} .
\end{array}
$$

Let $F(x, \lambda)=\left(F_{0}(x, \lambda), F_{1}(x, \lambda), \ldots, F_{n}(x, \lambda)\right)$ be defined by

$$
F_{i}(x, \lambda)=\sum_{k=0}^{m} A_{k} v_{i}\left(s_{k}, \lambda\right)
$$


It is clear that $F$ is continuous on $\bar{D} \times[0,1]$. We observe that a solution to the equation $F(x, \lambda)=q$ with $q$ defined by (7) corresponds to a Gauss formula for $C_{\lambda},\left(x_{*}, t_{*}\right)$, and $H_{n}$.

Lemma 8. If $x \in \partial D$, then $F(x, \lambda) \neq q$.

Proof. If $x \in \partial D$, one or more of the following must be true:

(i) $A_{i}=0$ or 1 for some $i=0,1, \ldots, m$.

(ii) $s_{i}=s_{i+1}$ for some $i=0,1, \ldots, m-1$.

(iii) $s_{m}=b$.

In any of the three cases, a solution to $F(x, \lambda)=q$ would imply the existence of an interpolation formula, having index smaller than $(n+1) / 2$, in contradiction to the Corallary to Lemma 5 (observe that if some $A_{i}=1$, then $A_{j}=0$ for all $j \neq i$, since $F_{0}(x, \lambda)=u_{0}\left(x_{*}, \lambda_{*}\right)$ means $\left.\Sigma_{k=0}^{m} A_{k}=1\right)$.

It follows that $\operatorname{deg}(F(\cdot, \lambda), D, q)$ is defined for $\lambda \in[0,1]$ and, moreover, is constant in $\lambda$. Hence, if we can show that $\operatorname{deg}(F(\cdot, 0), D, q) \neq 0$, property (iii) of degree will imply the existence of the desired formula for $C$.

Lemma 9. $\operatorname{Deg}(F(\cdot, 0), D, q) \neq 0$.

Proof. From Lemma 7 and the theory of Tchebycheff systems, there is a unique $x \in D$ such that $F(x, 0)=q$. We claim that $J F(x) \neq 0$.

$$
J F(x)=\operatorname{det}\left[\begin{array}{ccc}
v_{0}\left(s_{0}\right) & v_{0}\left(s_{1}\right) \cdots v_{0}\left(s_{m}\right) & A_{1} v_{0}^{\prime}\left(s_{1}\right) \cdots A_{m} v_{0}^{\prime}\left(s_{m}\right) \\
v_{1}\left(s_{0}\right) & v_{1}\left(s_{1}\right) \cdots v_{1}\left(s_{m}\right) & A_{1} v_{1}^{\prime}\left(s_{1}\right) \cdots A_{m} v_{1}^{\prime}\left(s_{m}\right) \\
\vdots & & \\
v_{n}\left(s_{0}\right) & v_{n}\left(s_{1}\right) \cdots v_{n}\left(s_{m}\right) & A_{1} v_{n}^{\prime}\left(s_{1}\right) \cdots A_{m} v_{n}^{\prime}\left(s_{m}\right)
\end{array}\right]
$$

We may factor out the positive $A_{i}$ 's, and the resulting determinant is nonzero since the $\left\{v_{k}(s)\right\}$ form an extended Tchebycheff system, by Lemma 6 . Hence, property (ii) of degree shows that $\operatorname{deg}(F(\cdot, 0), D, q)= \pm 1$.

The proofs for the remaining cases are similar. We will merely indicate the sets $D$ and the functions $F$ to be used.

Case (ii), $n=2 m$ and the point $\left(b, t_{*}\right)$ is involved.

$$
D=\left\{x=\left(A_{0}, \ldots, A_{m}, s_{0}, \ldots, s_{m-1}\right): 0<A_{i}<1, a<s_{0}<\cdots<s_{m}=b\right\} .
$$

$F(x, \lambda)$ is the same as before.

Case (iii), $n=2 m+1$ and $\left(b, t_{*}\right)$ is not involved.

$$
D=\left\{x=\left(A_{0}, \ldots, A_{m}, s_{0}, \ldots, s_{m}\right): 0<A_{i}<1, a<s_{0}<\cdots<s_{m}<b\right\} .
$$

$F(x, \lambda)$ is the same as before.

Case (iv), $n=2 m+1$ and $\left(b, t_{*}\right)$ is involved.

$$
\begin{gathered}
D=\left\{x=\left(A_{0}, \ldots, A_{m+1}, s_{1}, \ldots, s_{m}\right): 0<A_{i}<1,\right. \\
\left.a=s_{0}<s_{1}<\cdots<s_{m+1}=b\right\}, \\
F_{i}(x, \lambda)=\sum_{k=0}^{m+1} A_{k} v_{i}\left(s_{k}, \lambda\right), \quad i=0,1, \ldots, n .
\end{gathered}
$$


This completes the proof of statement (i) of Theorem 1. Statements (ii) and (iii) follow from Lemma 5 and its Corollary, respectively.

5. An Example. In his thesis [10], Shriver calculated several interpolation formulas, and then used them to approximate the solution to certain initial-boundary value problems for which the exact solution is known. We shall present his results for the following problem $[10$, p. 104]:

$$
\begin{gathered}
\frac{\partial u}{\partial t}=\frac{\partial^{2} u}{\partial x^{2}}, \quad-1<x<1, t>0 \\
u(-1, t)=u(1, t)=0 \\
u(x, 0)=100 \cos (\pi x / 2)
\end{gathered}
$$

Analytic solution:

$$
u(x, t)=100 e^{-(\pi / 2)^{2} t} \cos (\pi x / 2) .
$$

The $N$-point formulas (2) calculated had all points $\left(x_{i}, t_{i}\right)$ below the line $t=t_{*}$. Since the index of such formulas is $2 N$, they are exact for all heat polynomials of degree $2 N-1$ or less. The formulas were calculated by solving numerically the system of $2 N$ nonlinear equations

$$
\sum_{i=1}^{N} A_{i} u_{k}\left(x_{i}, t_{i}\right)=u_{k}\left(x_{*}, t_{*}\right), \quad k=0,1, \ldots, 2 N-1 .
$$

\begin{tabular}{|c|c|c|c|}
\hline$N$ & $A_{i}$ & $x_{i}$ & $t_{i}$ \\
\hline \multirow[t]{2}{*}{2} & .45883147 & -.74355958 & 0.0 \\
\hline & .54116853 & 1.0 & $.41717446(-1)$ \\
\hline \multirow[t]{3}{*}{3} & .22813315 & -1.0 & .11488151 \\
\hline & .37266462 & $.77632581(-1)$ & 0.0 \\
\hline & .39920223 & 1.0 & .19789729 \\
\hline \multirow[t]{4}{*}{4} & .16372791 & -1.0 & .18036319 \\
\hline & .27047010 & -.48382580 & 0.0 \\
\hline & .25078416 & .63467467 & 0.0 \\
\hline & .31501712 & 1.0 & .24771193 \\
\hline & .12458550 & -1.0 & .21452832 \\
\hline & 10204925 & -1.0 & $.26817914(-1)$ \\
\hline & .35485377 & $.22891853(-1)$ & 0.0 \\
\hline & .16530672 & 1.0 & $.22578853(-1)$ \\
\hline & .25320477 & 1.0 & .27498547 \\
\hline
\end{tabular}

In Table 1 we list formulas for the point $\left(x_{*}, t_{*}\right)=(.2, .4)$.

TABLE 1

Interpolation Formulas for $\left(x_{*}, t_{*}\right)=(.2, .4)$ 
TABLE 2

Approximate Solution of an Initial-Boundary Value Problem

$\begin{array}{ccccccc}\left(x_{*}, t_{*}\right): & (.1, .1) & (.2, .1) & (.3, .1) & (.1, .2) & (.2, .2) & (.3, .2) \\ \text { Exact Solution: } & 7.7172 & 7.4310 & 6.9610 & 6.0298 & 5.8062 & 5.4396 \\ N & & & & & & \\ 2 & 7.5884 & 7.2589 & 6.8005 & 5.3908 & 5.1908 & 4.8631 \\ 3 & 7.7261 & 7.4395 & 6.9691 & 6.0806 & 5.8527 & 5.4799 \\ 4 & 7.7171 & 7.4308 & 6.9616 & 6.0276 & 5.8040 & 5.4376 \\ 5 & 7.7172 & 7.4310 & 6.9618 & 6.0299 & 5.8063 & 5.4397 \\ & & & & & & \\ \left(x_{*}, t_{*}\right): & (.1, .3) & (.2, .3) & (.3, .3) & (.1, .4) & (.2, .4) & (.3, .4) \\ \text { Exact Solution: } & 4.7114 & 4.5366 & 4.2502 & 3.6812 & 3.5447 & 3.3209 \\ N & & & & & & \\ 2 & 3.4244 & 3.2974 & 3.2020 & 1.6304 & 1.7987 & 1.8694 \\ 3 & 4.8114 & 4.6334 & 4.3416 & 3.8415 & 3.6990 & 3.4654 \\ 4 & 4.7040 & 4.5295 & 4.2435 & 3.6643 & 3.3219 & 3.3057 \\ 5 & 4.7119 & 4.5371 & 4.2506 & 3.6828 & 3.5462 & 3.3224\end{array}$

In Table 2 we give the results of applying the formulas to the problem above. Five-point formulas are seen to produce 4 to 5 digit accuracy throughout the solution space.

Acknowledgment. The author wishes to thank Dr. Bruce Shriver for permission to include the above example in this paper.

Department of Mathematics

Texas A \& M University

College Station, Texas 77843

1. P. APPELL, "Sur l'équation $\partial^{2} z / x^{2}=\partial z / \partial t$ et la théorie de la chaleur," J. Math. Pures Appl., v. 8, 1892, pp. 187-216.

2. D. L. BARROW \& A. H. STROUD, "Existence of Gauss harmonic interpolation formulas," SIAM J. Numer. Anal. (To appear.)

3. D. COLTON, "The approximation of solutions to initial-boundary value problems for parabolic equations in one space variable," Quart. Appl. Math. (To appear.)

4. D. COLTON, "Complete families of solutions for parabolic equations with analytic coefficients," SIAM J. Math. Anal. (To appear.)

5. P. J. DAVIS \& M. W. WILSON, "Nonnegative interpolation formulas for uniformly elliptic equations," J. Approximation Theory, v. 1, 1968, pp. 374-380. MR 39 \#559.

6. S. KARLIN \& W. J. STUDDEN, Tchebycheff Systems: With Applications in Analysis and Statistics, Pure and Appl. Math., vol. 15, Interscience, New York, 1966. MR 34 \#457.

7. J. M. ORTEGA \& W. C. RHEINBOLDT, Iterative Solution of Nonlinear Equations in Several Variables, Academic Press, New York and London, 1970. MR 42 \#8686.

8. M. H. PROTTER \& H. F. WEINBERGER, Maximum Principles in Differential Equations, Prentice-Hall, Englewood Cliffs, N. J., 1967. MR 36 \#2935.

9. P. C. ROSENBLOOM \& D. V. WIDDER, "Expansions in terms of heat polynomials and associated functions," Trans. Amer. Math. Soc., v. 92, 1959, pp. 220-266. MR 21 \#5845.

10. B. D. SHRIVER, Interpolation Formulas of Gauss Type for Approximate Solution of the n-Dimensional Heat Equation, Ph.D. Thesis, State University of New York at Buffalo, 1971.

11. J. T. SCHWARTZ, Nonlinear Functional Analysis, Gordon and Breach, New York, 1969. 
12. A. H. STROUD, "Gauss harmonic interpolation formulas," Comm. Assoc. Comput. Mach., v. 17, 1974, pp. 471-475.

13. A. H. STROUD, "Some interpolation formulas for the Neumann problem for the $n$ sphere," SIAM J. Numer. Anal. (To appear.)

14. A. H. STROUD \& D. L. BARROW, "Gauss formulas' for the Dirichlet problem," Bull. Amer. Math. Soc., v. 80, 1974, p. 1230.

15. D. V. WIDDER, “Analytic solutions of the heat equation," Duke Math. J., v. 29, 1962, pp. 497-503. MR 28 \#364. 